Br Heart $\mathcal{F}$ 1985; 53: 577-84

\title{
Correspondence
}

\section{Left ventricular function in coronary artery disease}

Sir,

I write concerning the article by Lahiri et al on the "Assessment of left ventricular function in coronary artery disease with the nuclear probe during intervention studies" (1984; 52: 422-30). The data on ejection fraction changes during effort is interesting. There is, however, a problem with the method of determining filling and ejection rates. Ejection and filling rates are calculated by the probe according to the formula:

$$
\frac{\mathrm{CT} 3-\mathrm{CT} 2}{\mathrm{~T} 3-\mathrm{T} 2} \times \frac{1}{\mathrm{Cn}}
$$

where CT3 is the counts at cursor position T3, CT2 counts at cursor T2 (T3 and T2 represent the time positions of the T3 and T2 cursors), and $\mathrm{Cn}$ the normalisation counts. Normalisation counts equal CT1 minus BKG when $T 1$ is not equal to 0 , and when $T 1$ is equal to $0 \mathrm{Cn}$ equal to $B K G$. When $T 1$ is positioned at the midpoint between $T 2$ and $T 3$, ejection rate will be normalised to average counts. When $\mathrm{T} 1$ is positioned at the end diastolic counts filling rate will be normalised to end diastolic volume. Both exercise and the administration of nitrates may, however, be expected to change ventricular volume. If ventricular volume changes then the normalisation counts will change producing apparent changes in filling or ejection rate not reflecting true changes in these variables. To deal with this problem the T1 cursor should be positioned at 0 and normalisation will then be to background counts. Under these circumstances changes in filling or ejection rate may be followed independently of changes in ventricular volume. As things stand it may be thought that no reliance can be placed on quoted changes in filling or ejection rate.

David Robson, Greenwich District Hospital, London SE10 9HE.

This letter was shown to the authors, who reply as follows:

\section{Sir,}

We have carefully considered Dr Robson's comments about our paper $(1984 ; 52: 422-30)$. In reply to his comments we would like to establish certain facts.
Firstly, the measurement of slopes from the left ventricular time-activity curves by the computer of the nuclear stethoscope is based on relative rather than absolute left ventricular volumes. The methods used for calculating peak filling rate are based on those described by previous authors - namely, Bonow et $a l,{ }^{1}$ Reduto et al, ${ }^{2}$ and Mancini et al, ${ }^{3}$ and these have been quoted in the text.

Secondly, in patients with obstructive coronary artery disease, during exercise induced myocardial ischaemia, the following changes occur: changes in left ventricular volume, increases in left ventricular end diastolic pressure, left ventricular wall tension, left atrial pressure, and increased cardiopulmonary blood volume and increased pulmonary wedge pressure. ${ }^{4}$

Thus exercise induced ischaemia would affect both left ventricular end diastolic volume and background counts (which represents cardiopulmonary blood volume). Nitrates therefore would have a reverse effect on both these variables. Thus, in our opinion, the correction or normalisation of the slope to end diastolic volume or background would have the same errors as indicated by Dr Robson. We have, in fact, applied both of these corrections to the peak filling rate in previous experiments and found that the direction of changes is similar. We do agree, however, that during intervention studies using the nuclear stethoscope or the gammacamera the values of the rates of filling and emptying are relative unless one is able to perform attenuation correction and convert the relative data into absolute volumes.

Thus it follows that "normalisation to background counts allow changes in filling or ejection rate to be followed independently of changes in ventricular volume" is manifestly incorrect since all the volume variables change during interventions with exercise or with nitrates in patients with obstructive coronary artery disease.
A Lahiri,
E B Raftery,
Department of Cardiology,
Northwick Park Hospital,
Harrow,
Middlesex HA1 3UJ. 


\section{References}

1 Bonow RO, Bacharach SL, Green MV, et al. Impaired left ventricular diastolic filling in patients with coronary artery disease: assessment with radionuclide angiography. Circulation 1981; 64: 315-23.

2 Reduto LA, Wickemeyer WJ, Young JB, et al. Left ventricular diastolic performance at rest and during exercise in patients with coronary artery disease. Assessment with first-pass radionuclide angiography. Circulation 1981; 63: 1228-37.

\section{Analysis of circadian blood pressure rhythms}

Sir,

We read with interest the observation by Davies et al (1984; 52: 93-8) on the circadian rhythm of blood pressure. The authors state that Millar-Craig et al reported a second peak of blood pressure in the early evening. ${ }^{1}$ We consider that this is not a correct citation, as Millar-Craig et al reported that ". . . blood pressure fell progressively during the day and rose in the early hours of the morning."

Davies et al mention the difficulties in quantifying variations in the blood pressure. Some of the basic work on rhythmic blood pressure changes (summarised in ${ }^{2}$ ) deals with the problems of recording, reproducibility, and analysis of blood pressure patterns. Using cosinor analysis, first introduced by Halberg et $\mathrm{al}^{3}$ might improve the accuracy of analysis. It provides an exact calculation of rhythm variables such as mesor, amplitude, and acrophase of circadian rhythmic changes. ${ }^{4-6}$ It would be interesting to evaluate the carefully documented data of Davies et al by cosinor analysis. We wonder whether this method of analysis would confirm the reported two hour difference between the nadir of the paced group and the control group.

The authors also point out that the amplitude of the cirdacian pattern was lower in the paced than in the hypertensive group. Judging from the data in Fig. 1 of their article this seems not unlikely. Quantitative evaluation of the amplitudes, however, is not reported but might be done by cosinor analysis. Furthermore, cosinor analysis might allow a better comparison of circadian heart rate and blood pressure variations.

Alexander L Gerbes, *

Ekkehard Haen, $t$

Bernhard Arbogast,

^ Medizinische Klinik II,

Klinikum Grosshadern der Universität Munchen.

†Institute of Pharmacology,

Universität Munchen.

$¥$ Bavarian Reinsurance Company, D-8000 Munich 22,

Federal Republic of Germany.
3 Mancini GBJ, Slutsky RA, Norris SL, Bhargava V, Ashburn WL, Higgins CB. Radionuclide analysis of peak filling rate, filling fraction and time to peak filling rate. Response to supine bicycle exercise in normal subjects and patients with coronary disease. Am $\mathcal{F}$ Cardiol 1981; 51: 43-51.

4 Nichols AB, Strauss HW, Moore RH, et al. Acute changes in cardiopulmonary blood volume during upright exercise stress testing in patients with coronary heart disease. Circulation 1979; 60: 520-30.

\section{References}

1 Millar-Craig MW, Bishop CN, Raftery EB. Circadian variation of blood-pressure. Lancet 1978; i: 795-7.

2 Halberg F. Quo vadis basic and clinical chronobiology: promise for health maintenance. Am $\mathcal{F}$ Anat 1983; 168: 543-94.

3 Halberg F, Tong YL, Johnson EA. Circadian system phase -an aspect of temporal morphology; procedures and illustrative examples. In: Von Mayersbach $\mathrm{H}$, ed. The cellular aspect of biorhythms. Berlin: Springer, 1967: 20-48.

4 Bingham C, Arbogast B, Guillaume GC, Lee JK, Halberg $F$. Inferential statistical methods for estimating and comparing cosinor parameters. Chronobiologia 1982; 11: 397-439.

5 Gerbes AL, Arbogast B, Schick P, Messerschmidt O. Acute radiation injury of mice and the influence of sudden time shift. Radiat Res 1984; 99: 285-93.

6 Haen E, Halberg F, Cornelissen G. Cortisol marker rhythmometry in pediatrics and clinical pharmacology. Annual Review of Chronopharmacology 1984, in press.

This letter was shown to the authors, who reply as follows:

Sir,

We thank Drs Gerbes et al for their interest in our paper on circadian rhythms of blood pressure in paced patients. They correctly cite our original description of the broad 24 hour pattern of blood pressure ${ }^{1}$; however, most of our data on closer inspection showed a subsidiary late afternoon rise of lower amplitude than that seen after waking. This is visible in Figs. 1 and 3 of the original publication. ${ }^{1}$

The problems inherent in describing ambulatory intra-arterial blood pressure patterns have been recognised by several groups of workers. We find it difficult to accept that Halberg's cosinor analysis would be able to provide sufficient accuracy in this case. As we understand it, the technique consists of fitting a single 24 hour cosine curve to the data using the method of least squares. Variables such as the amplitude, mesor (average value), and acrophase (tim- 